Japanese Psychological Research

1978 , Vol. 20, No $4,167-176$

\title{
THE EFFEGTS OF DOUBLE-ALTERNATION SCHEDULES OF SHOCK INTENSITY UPON PATTERNING OF SUPPRESSION OF LICKING IN RATS WITH SPECIAL REFERENCE TO CUE-UTILIZATION ${ }^{1}$
}

\author{
HIROSHI IMADA, KOZO SUGIOKA 2 , YUJI OHKI, \\ HAJIME NINOHIRA AND AKIHIKO YAMAZAKI \\ Department of Psychology, Kwansei Gakuin University
}

\begin{abstract}
Thirsty rats were run in a water-drinking situation in which brief shocks of two different intensities were given with a fixed-time 40 -sec schedule and in a doublealternation (DA) pattern of shock intensity, without any signal in Experiment I and with two different kinds of signal during the shock-shock interval in Experiment II. The three groups in Experiment II differed regarding the relationship of the signal with shock intensities. In Group SA, tone and light were presented in single-alternation and in Group $R$ they were presented in a random order. In all the above groups there was no evidence of DA patterning of the suppression in licking to correspond with the variation in shock intensity. Only in Group DA, in which the two modalitics of signal corresponded with the two respective shock intensities, was a DA patterning of suppression observed. There was clear evidence in all groups that the strong shock had an immediate burst-inducing function. In Experiment III the possibility that rats would predict forthcoming events on the basis of shock-cues was pursued but the results turned out to be negative.
\end{abstract}

Imada and Okamura (1975) have found that when brief electric shocks were presented to thirsty rats with an FT45-sec schedule during 5-min water-drinking sessions, rats' licking behavior came to be gradually suppressed as the time of the next shock approached. In addition to this evidence of temporal conditioning, they also observed a clear single-alternation patterning of suppression in licking when the shock intensity was made strong and weak in an alternate order. More specifically, rats' licking rate was lower during the 45-sec intervals which ended up with the stronger shock (or during the $\mathrm{S}$ intervals) than during those which terminated with the weaker shock (or during the $\mathrm{W}$ intervals). The evidence was also provided

1 The present study was supported by a Grant in Aid for Scientific Researches (No. 051085 and No. 245016), Ministry of Education, Japan.

2 Now at Department of Anatomy, Shimane Medical University, Izumo, Japan. which showed that less suppression of licking during the $\mathrm{W}$ intervals than during the $S$ intervals was not due to unconditional aggression-like bursts of responding to the strong shock they had just received, but due to the rats' expecting the weaker shock at the end of the $W$ intervals.

The authors discussed the above facts in terms of fear conditioned to various invensities of trace of shocks. To quote: "The trace to which fear was conditioned the least should have been that immediately after the stronger shock because it was most remote from the weaker shock. The trace to which fear was conditioned the most should have been that during Period 9 (or the last of the nine 5 -sec periods of the $45-\mathrm{sec}$ interval) of the $\mathrm{S}$ intervals because it was most closely associated with the stronger shock. Between these two extremes, one can assume a continuum of intensity of traces of shock, to each value of which different strengths of fear 
would have been conditioned" (Imada \& Okamura, 1975, p. 224).

\section{EXPERIMENT I}

If the above interpretation of the facts is correct, a double-alternation patterning of suppression in licking can hardly be expected when the shock intensity is made stronger and weaker according to a double-alternation (DA) schedule. It is because the stimulus trace of the stronger (or weaker) shock is followed by the stronger shock half of the times and by the weaker shock the other half of the times. In other words, there is no consistent relationship, as in the case of a singlealternation (SA) schedule, between the intensity of the shock-trace and the intensity of the shock which is to follow it. Experiment I was a single group experiment designed simply to test the above hypothesis, which also served as a stepping stone to move on to Experiment II.

\section{Method}

Subjects. The subjects were 10 experimentally naive male albino rats of the Wistar strain. They weighed between 192-240 g (mean: $207.5 \mathrm{~g}$ ) at the beginning of the experiment.

Apparatus. The apparatus and the general experimental set-up were exactly the same as in the previous study (Imada \& Okamura, 1975). Briefly, the apparatus was a drinking box $(20 \mathrm{~cm}$ long, $10 \mathrm{~cm}$ wide and $15 \mathrm{~cm}$ high) with a grid floor. A thirsty rat could drink water by licking at the tip of the drinking tube through a hole at one narrow end of the box and the number of rat's tongue-licks was measured and recorded automatically. The scrambled electric shock was delivered from the ac-shock source to the grid floor through a $250 \mathrm{k} \Omega$ current-limiting resistor in series with the rat. Four rats, at maximum, were run simultaneously by using four identical drinking boxes and the experiment was run under a dimly lit condition $(.5 \mathrm{l} x)$ and with continuous masking noise of $64 \mathrm{~dB}$ (c) produced by an electric fan.
Procedure. [Preliminary training of drinking (22 days)] Following 5 days of handling the rats were trained to drink water from the tube in the drinking box for 22 days, $5 \mathrm{~min}$ per day. Water was made available to the rats for $1 \mathrm{hr}$ after a daily session. On the 22nd day of training, the mean number of licks in 5-min session was 1535.0 .

[Shock training: Phase 1 (5 days)] For the rest of the training, an electric shock of $.7-\mathrm{sec}$ duration was presented eight times per session on an FT40-sec schedule. The first shock was invariably given $10 \mathrm{sec}$ after the start of each session. All the shocks were of $90 \mathrm{~V}$ $(.28 \mathrm{~mA})^{3}$ intensity during this initial phase.

[Shock training: Phase 2 (28 days)] Throughout the rest of the training, the shock intensity was made weak and strong according to the DA schedule. On odd numbered days, the shock sequence started with a weak shock, and on even numbered days, with a stronger shock. The weaker of the shocks was kept at $90 \mathrm{~V}(.28 \mathrm{~mA})$ throughout the experiment. The stronger shock was gradually increased in the following order: $120 \mathrm{~V}(.37 \mathrm{~mA})$ (4 days), $150 \mathrm{~V}(.47 \mathrm{~mA})(8$ days) and $170 \mathrm{~V}(.53 \mathrm{~mA})$ (16 days).

In the descriptions to follow, the shockshock intervals will be referred to as "intervals" and each of the 5-sec periods within each interval as "period." The number of licks was printed out in 5-sec period. The intervals terminating with the stronger shock will be referred to as $S$ intervals and those with the weaker shock as $\mathrm{W}$ intervals. There were two kinds of $\mathrm{S}$ intervals, the one beginning with the strong shock and the other with the weak shock and they will be designated as $\mathrm{sS}$ and $w S$ intervals, respectively. In the same way, there were two kinds of $\mathrm{W}$ intervals and they will be referred to as $s W$ and $w W$.

\section{Results and Discussion}

The primary result of Experiment I was that a DA patterning of suppression of drinking was not observed. Table 1

${ }^{3}$ In the present paper, all the values of $\mathrm{mA}$ were estimated by assuming the resistance of a rat to be $100 \mathrm{k} \Omega$. 
TABLE 1

Mean number of licks per $5 \mathrm{sec}$ for four different kinds of intervals. The results are for the last 4 days of training where the shock intensities were $90 \mathrm{~V}$ and $170 \mathrm{~V}$

\begin{tabular}{|c|c|c|c|}
\hline \multirow[b]{2}{*}{$w$} & W & S & \multirow[b]{2}{*}{13.36} \\
\hline & 12.60 & 14.11 & \\
\hline s & 14.67 & 16.72 & 15.70 \\
\hline & 13.64 & $15 . t^{\prime \prime 2}$ & \\
\hline
\end{tabular}

summarizes the mean number of licks per $5 \mathrm{sec}$ for the four different kinds of intervals during the last four days of training where the strong and weak shocks were $170 \mathrm{~V}$ and $90 \mathrm{~V}$, respectively. The small letters indicate the intensity of the shock with which the interval began and the capital letters indicate that with which the interval ended. The column means, then, show the effect of the expected shock intensity upon licking and the row means the aftereffect of the two intensities of shock on licking. Both main effects were significant (W vs. $\mathrm{S} ; F=6.21, d f=1 / 9$, $p<.05:$ w vs. $s ; F=5.26, d f=1 / 9, p<$ $.05)$. The interaction effect of the above two terms was not significant $(F<1)$.

The results as a whole thus indicated: 1) The rats licked significantly more when the stronger shock, rather than the weaker shock, was given at the end of the interval. 2) The rats licked significantly more during the intervals which started with the stronger shock rather than with the weaker shock. Discussions on the implications of these facts as well as references to the detailed fact regarding temporal conditioning which are omitted here, will be made in conjunction with the discussion of the results of Experiment II.

Turning back to the hypothesis of peripheral nature put forward in the introduction section, the fact that the rats could not show the DA patterning of suppression in licking corresponding to the DA pattern of shock intensity does not contradict with the hypothesis although it does not positively support it.

\section{EXPERIMENT II}

Although the results of Experiment I were those which had been theoretically expected, they were contrary to the implicit expectation of the present authors who had faintly hoped that the rats might be 'intelligent enough to recognize' the DA pattern of shock intensity. In Experiment II, then, efforts were made to obtain the DA patterning in licking under the DA pattern of shock intensity by arranging the experimental conditions in such a way that the rats, in theory, should show it.

It is of interest to note that in an instrumental reward learning situation, rats failed to show DA patterning behavior under DA pattern of reward (R) and nonreward (NR), in spite of the fact that they successfully showed appropriate alternate fast-and-slow running under a $\mathrm{SA}$ pattern of R and NR (e.g., Bloom \& Capaldi, 1961). Recently, however, Capaldi (1971) and Pavlik and Collier (1975) have reported that rats given a DA schedule ( $R, R, N R, N R, R, R, \ldots)$ in which the first $R$ and first $N R$ of each pair occurred in a black (B) alley and the second $\mathrm{R}$ and NR of each pair occurred in a white (W) alley, developed accurate patterning behavior. Pavlik and Collier interpreted this so-called "signaled DA patterning" phenomenon by identifying four different compounds of stimuli, two of which were consistently followed by $R$ and the other two by NR. More specifically, the relationships between four stimulus compounds and $\mathrm{R}$ and $\mathrm{NR}$ are as follows, where $B$ and $W$ represent black and white external cues, respectively, and $s^{R}$ and $s^{\mathrm{NR}}$ represent traces of $\mathrm{R}$ and $\mathrm{NR}$, respectively.

$$
\begin{gathered}
s^{\mathrm{R}}+\mathrm{B}: \mathrm{NR}, \mathrm{s}^{\mathrm{NR}}+\mathrm{W}: \mathrm{NR}, \\
\mathrm{s}^{\mathrm{NR}}+\mathrm{B}: \mathrm{R}, \mathrm{s}^{\mathrm{R}}+\mathrm{W}: \mathrm{R}
\end{gathered}
$$

In Experiment II, an attempt was made to apply the Pavlik and Collier assumption to Experiment I type of situation. 
Two modalities of stimuli, or tone and light, wcre used as external cues. Then, during the first of the two successive $S$ and the two successive $W$ intervals, tone ( $T$ ) was presented and during the second of the two successive $\mathrm{S}$ and the two successive $\mathrm{W}$ intervals, the light $\left(\mathrm{L}_{\mathrm{H}}\right.$ ) was presented. The cue was given for the last $35 \mathrm{sec}$ of the 40-sec intervals. The relationships between the intensity of the shock and four stimulus compounds preceding the shocks were as follows, where $s$ and $w$ represented the traces of the strong and weak shocks, respectively.

$$
\begin{aligned}
& s+\mathrm{L}: \mathrm{S}, \mathrm{s}+\mathrm{T}: \mathrm{W}, \\
& \mathrm{w}+\mathrm{L}: \mathrm{W}, \mathrm{w}+\mathrm{T}: \mathrm{S}
\end{aligned}
$$

In the presence of $s+T$ and $w+L$, more licking should be expected than in the presence of $s+L$ and $w+T$. In other words a $\mathrm{DA}$ patterning of suppression in licking should appear under such a condition.

\section{Method}

Subjects. The subjects were 38 experimentally naive male albino rats of the Wistar strain and they weighed between $220-260 \mathrm{~g}$ (mean: $239.3 \mathrm{~g}$ ) at the beginning of the experiment.

Apporatus. The apparatus was exactly the same as that of Experiment I. Two kinds of stimuli, light and tone, wcre used as signals in Experiment II. The light was presented by the $100 \mathrm{~W}$ lamp suspended approximately $163 \mathrm{~cm}$ above the apparatus. Turning on this lamp increased the illumination inside the drinking box from $.5 \mathrm{~lx}$ to $23 \mathrm{~lx}$. The tone,
$1000 \mathrm{~Hz}$ and $83 \mathrm{~dB}(\mathrm{c})$, was presented through a speaker suspended $200 \mathrm{~cm}$ above the apparatus.

Procedure. [Preliminary training of drinking (40 days)] Following 5 days of handling the rats were trained to drink water from the tube 5 min per day in exactly the same way as in Experiment I. On the 37 th day of training, the subjects were divided into three groups matched on the basis of both the body weight and the mean number of licks on the training days 34-37. The mean number of licks per session of these four days for all rats was 1481.2.

[Shock training (42 days)] Excepting that the light or tone was presented for $35 \mathrm{sec}$ during cach interval, the procedure was the same as in Experiment I. The strong and weak shocks $(.7 \mathrm{sec})$ were presented with a DA and FT40-sec schedule. On odd numbered days the session started with the weak shock and on even numbered days with the strong shock. The weaker of the shocks was kept at $90 \mathrm{~V}$ $(.28 \mathrm{~mA})$ throughout the experiment, but the stronger shock was gradually increased in the following order: $110 \mathrm{~V}(.34 \mathrm{~mA})$ (6 days), $130 \mathrm{~V}(.40 \mathrm{~mA})(6$ days $), 150 \mathrm{~V}(.47 \mathrm{~mA})(6$ days) and $170 \mathrm{~V}(.53 \mathrm{~mA})(24$ days $)$. Shock schedules were exactly the same for all groups.

Three groups differed with respect to the manner two kinds of signal, tone $(\mathrm{T})$ and light (L), were presented. The signals were presented for the last $35 \mathrm{sec}$ of 40 -sec intervals. In Group SA (or single-alternated signal group; $n=14) \mathrm{L}$ and $\mathrm{T}$ were presented alternately as shown in Fig. 1. In this group, four kinds of stimulus compound consisting of the trace

\begin{tabular}{|c|c|c|c|c|c|c|c|}
\hline INTERV & ALS & 1 & 2 & 3 & 4 & 5 & 7 \\
\hline & 5 & & & & & & \\
\hline GROUP & SA & $\mathbf{L}$ & $T$ & & $T$ & L & L \\
\hline GROUP & DA & $\mathbf{L}$ & $T$ & 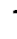 & L & L & $T$ \\
\hline GROUP & $\mathbf{R}$ & $L$ & $T$ & & $L$ & $T^{T}$ & L \\
\hline
\end{tabular}
of the preceding shock and the external signal

Fig. 1. An example of DA schedule of shock intensity which started with the strong shock. At the point of triangles, either strong (S) or weak (W) shock was presented. Light (L) and Tone (T) were presented to three groups as shown. 
(or $\mathrm{s}+\mathrm{L}, \mathrm{s}+\mathrm{T}, \mathrm{w}+\mathrm{L}$ and $\mathrm{w}+\mathrm{T}$ ) related consistently with the shock intensity followed (see the introduction section of Experiment II). For half of the subjects the session always started with $L$ and for the other half with $T$.

In Group DA (or double-altemated signal group ; $n=12) \mathrm{L}$ and $\mathrm{T}$ were presented according to a $\mathrm{DA}$ schedule. In this group there was a systematic relationship between the external signal and the shock intensity; i.e., L (or T) always preceded the weak shock and $\mathrm{T}$ (or $\mathrm{L}$ ) the strong shock as shown in Fig. 1. In Group $\mathrm{R}$ (or random signal group; $n=12$ ) the relationship between the signal and the shock intensity was made random. For all three groups no signal was presented preceding the first shock of each session.

\section{Results and Discussion}

A set of tables in Table 2 summarizes the mean number of licks per $5 \mathrm{sec}$ for the last four intervals of each session during the last eight days of training where the strong and weak shocks were $170 \mathrm{~V}$ and $90 \mathrm{~V}$, respectively. Figures 2 and 3 represent the changes of the licking rate as a function of periods within intervals for four different kinds of intervals separately. The graphs are for Group SA and DA and the graph for Group $R$ is omitted because the general picture did not differ from that shown in Fig. 2.

As is obvious from Figs. 2 and 3, there is clear evidence of temporal conditioning in Groups SA and DA and also, although not graphically shown, in Group $R$. The rats' licking rate progressively decreased as the time of the next shock approached. This evidence is in line with the facts obtained by Hendry, Yarczower, and

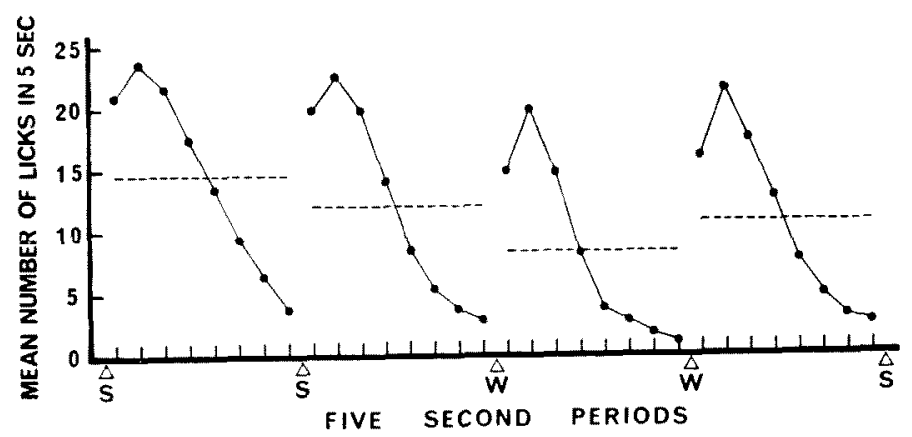

Fig. 2. Mean number of licks as a function of periods within four different kinds of intervals of Group SA. Values shown in Table $2 \mathrm{~A}$ are represented by the broken lines.

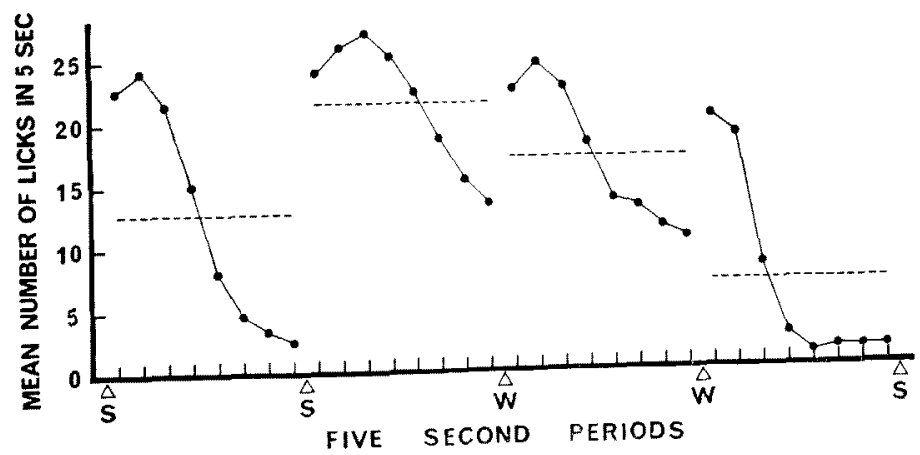

Fro. 3. Mean number of licks as a function of periods within four different kinds of intervals of Group DA. Values shown in Table $2 B$ are represented by the broken lines. 
TABLE 2

Mean number of licks per 5 sec for four different kinds of intervals for three groups separately. The results are for the last 4 intervals of each session for the last 8 days of training where the shock intensities werc $90 \mathrm{~V}$ and $170 \mathrm{~V}$

A

\begin{tabular}{|c|c|c|}
\hline \multicolumn{3}{|c|}{ Group SA } \\
\hline & $W$ & $\mathrm{~S}$ \\
\hline & 8.32 & 10.68 \\
\hline & 12.01 & 14.59 \\
\hline & 10.17 & 12.64 \\
\hline
\end{tabular}

W vs. S: $F_{1,13}=11.20^{*}$

w vs. $s: F_{1,13}=19.34 * * *$

WS $\times$ ws : $F<1$
B

\begin{tabular}{|c|c|c|}
\hline \multicolumn{3}{|c|}{ Group DA } \\
\hline W & $\mathrm{S}$ & \\
\hline 16.95 & 6.85 & 11.90 \\
\hline 21.40 & 12.75 & 17.08 \\
\hline 19.18 & 9.80 & \\
\hline $\begin{array}{l}F_{1} \\
F_{1} \\
F_{1}\end{array}$ & $\begin{array}{l}=43.32^{*} \\
=69.94 \\
=2.20\end{array}$ & \\
\hline
\end{tabular}

$\mathrm{C}$

\begin{tabular}{|c|c|c|}
\hline \multicolumn{3}{|c|}{ Group R } \\
\hline W & S & \\
\hline 12.13 & 12.30 & 12.22 \\
\hline 13.55 & 17.34 & 15.45 \\
\hline 12.84 & 14.82 & \\
\hline $\begin{array}{l}F_{1}, \\
F_{1} \\
F_{1}\end{array}$ & $\begin{array}{l}14.19 \\
=20.71 \\
=13.87\end{array}$ & \\
\hline
\end{tabular}

Switalski (1969), Imada and Okamura (1975) and also with the results obtained in Experiment I.

In Group DA, in which there were consistent relationships between the intensity of the shock and the external signal, there was clear evidence of discrimination and the rats licked significantly more during the two $\mathrm{W}$ intervals than during the two $\mathrm{S}$ intervals. This is a case of simple discrimination in which the discriminanda were external cues.

The failure to obtain DA patterning of suppression in Group SA was in contradiction with the theory put forward in the introduction section of Experiment II. More specifically, there was no indication in the results that rats discriminated the stimulus compounds which consistently preceded the strong shock (e.g., s+L and $w+T)$ and those which consistently preceded the weak shock (e.g., s+T and $w+$ L). Comparison of Table $2 \mathrm{~A}$ and Table 1 indicates that the addition of the external signals in SA had no function in altering the patterns of licking. Although the results of Group $R$ were generally in line with those of Group SA and of Experiment I, one difference was that in Group $R$, there was significant interaction of W-S factor and w-s factor as shown at the lower part of Table 2. The meaning of the presence of this interaction effect in
Group $\mathrm{R}$ and its absence in other groups is not clear.

\section{General Discussion of EXPERIMENTS I AND II}

One characteristic which was common to all four groups of rats of Experiments I and II was the significant main effect of the preceding shock intensity: The rats licked significantly more following a strong shock than following a weak shock. This effect is in line with the findings by Amsel and Maltzman (1950), Siegel and Brantley (1951) and Ullman (1951). In the first two of these studies rats' consummatory behavior measured immediately after the shock administered in different situation was facilitated and the authors, on the basis of the drive theory, interpreted the fact in terms of the energizing aftereffect of an electric shock. In Ullman's study, rats' eating behavior was facilitated when the shock was given than when it was not given. Further the extent to which the facilitation of eating occurred was greater with the strong shock than with the weak shock. The phenomenon was referred to as compulsive eating by Ullman (1951). In a more recent study by Hutchinson and Emley (1971) a monkey sitting in a monkey chair bit a rubber hose suspended in front of its face after each shock ad- 
ministration, which they referred to as biting attack behavior. They also showed that this biting attack was more frequent following stronger shocks than following weaker shocks. Response-bursts after shocks were also observed in conditioned suppression experiments (Estes \& Skinner, 1941 ; Weiss \& Strongman, 1969). Some of these results may be explained, as Davis, Memmott, and Hurwitz (1975, p. 183) attempted, in terms of "autocontingency," ascribing the bursts of responding after each shock to the presence of shock-free or safety interval after the shock. The present fact of greater burst-inducing power of the strong shock over the weak one however, cannot be explained solely in the above manner because in the present experiments both strong and weak shocks predicted a shock-free interval of $40 \mathrm{sec}$. It is of interest to note that the facilitating aftereffect of strong shock upon licking was superimposed upon appropriate shock-intensity discrimination observed in Group DA as shown in Fig. 3.

What further needs to be explained is the fact that in Groups $S A$ and $R$ of Experiment II and also in Experiment I, the rats licked significantly more during the $S$ intervals than during the $\mathrm{W}$ intervals. This puzzling fact seems to be best explained by assuming a suppressive effect that persisting emotionality would have had upon drinking in addition to the immediate facilitation effect of the shock upon licking. Although a posteriori in nature, the reasoning may go as follows: The effect of the receipt of strong shock two in succession (with a 40-sec interval between shocks in the present case) would accumulate and come to have disrupting effect on behavior. The receipt of the weak shock two in succession, on the other hand, would lead to the recovery from disruption. Combining this assumption with the immediate energizing effect of the strong shock upon behavior, one may explain the pattern in the changes of licking rate represented by the broken lines of Fig. 2. During the sS interval, the cumulative effect of shocks may be small and the strong shock would exert its encrgizing effect. During the sW interval, an immediate energizing effect may be present as in the case of the $\mathrm{sS}$ but the disrupting effect of two successive strong shocks may become evident during this interval. During the intervals beginning with the weaker shock, the energizing effect of shock may be present to the lesser extent than during the intervals beginning with the strong shock and the rate of licking may be determined by the level of then persisting emotional level of rats. Since, by assumption, the receipt of weak shock two in succession may lead to recovery from suppression, the licking rate during wS intervals is expected to be higher than that during $w W$ intervals. Since, according to the above assumptions, the rats are expected to lick more during $\mathrm{sS}$ intervals than during $s W$ intervals and during $w S$ intervals than during $w W$ intervals, the fact of significantly higher rate of licking during $\mathrm{S}$ intervals as a whole in Groups SA and $R$ may thus be understandable. It should, however, be noted that when the intensity of shock to receive next was appropriately predicted by the signal as in Group DA, the rats' licking rate was more under the control of the discriminanda or signals than under the control of persisting emotional level (see Fig. 3).

\section{EXPERIMENT III}

There are at least two possible ways to develop the present series of experiments : One is to empirically test the adequacy of the assumption just described on the possible interaction of the immediate energizing effect of the strong shock and the cumulative effect of shocks upon behavior. The other is to further test whether or not rats could predict the forthcoming event on the basis of the cue provided by the shock without being confounded with the immediate energizing effect of the 
strong shock upon behavior. Since this second problem has been the primary concern of the present authors in running the present experiments, the Experiment III was designed to investigate this second problem.

In this experiment, two pulses of shock of $0.2 \mathrm{sec}$ each was presented in succession with the inter-pulse interval of $0.6 \mathrm{sec}$, which comprised one "shock." One of the two pulses was of weak intensity and the other of strong intensity and each shock was either of strong-weak (SW) or of weak-strong (WS) order. Since the total amount of aversive experience was the same at each "shock," the possible cue function of the shock can be investigated without being confounded with the differential energizing functions of different intensities of shock. The event to be predicted by the shock cues was the length of shock-free interval. Past experiments on conditioned suppression have shown that the overall rate of rats' operant or consummatory behavior was suppressed less when the minimum inter-shock interval was long than when it was short, indicating the rats' ability to use temporal cues as predictors of danger and safety (Seligman, 1968; Nageishi \& Imada, 1974).

Thus the purpose of the present experiment was to study the possibility that the rats would be able to use the different shock patterns as predictors of long or short shock-free interval using the suppression technique as used in Experiments I and II.

\section{Method}

Subjects. The subjects were 12 experimentally naive male albino rats of Wistar strain weighing between 266-378 g (mean: $344.3 \mathrm{~g}$ ) at the beginning of the experiment.

Apparatus. The apparatus was the same as in Experiments I and II.

Procedure. The maintenance schedule and the procedure for the preliminary training of drinking were the same as in Experiments I and II. The mean number of licks during 5-min session on the final no-shock day was 1572.4.

On the shock days, six shocks were superimposed upon licking during each session, three of the shocks were of WS pattern and the other three of SW pattern (see the introduction section of Experiment III). The first shock was invariably given $65 \mathrm{sec}$ after the start of each session. For half of the rats the WS pattern was followed by the 60 -sec shock-free interval and the SW pattern by the 15-sec shock-free interval. For the other half, the above relations were reversed and hence the relations were WS-short-safety and SW-longsafety. The following four shock sequences were prepared and used in rotation, so that each sequence appeared every four days. Sequence 1; WS, SW, WS, SW, WS, SW: Sequence 2 ; SW, WS, WS, SW, SW, WS: Sequence 3 ; SW, WS, SW, WS, SW, WS : Sequence 4 ; WS, SW, SW, WS, WS, SW. For half of the rats the WS and SW of each of the above sequences were reversed. More concretely, for half of the rats the first shock day (Sequence 1) started with WS-long-safety and for the other half with $\mathrm{SW}$-long-safety. The intensities of weak and strong pulses were $70 \mathrm{~V}(.22 \mathrm{~mA})$ and $140 \mathrm{~V}(.43 \mathrm{~mA})$, respectively, for the first 16 days but they were increased to $80 \mathrm{~V}$ $(.25 \mathrm{~mA})$ and $160 \mathrm{~V}(.50 \mathrm{~mA})$ for the last six days. It had been ascertained by the preliminary observation that the vigorousness of rats, behavior to these paired pulses of different intensities was discriminably different.

\section{Results and Discussion}

The mean values for the number of licks per $5 \mathrm{sec}$ during the initial $15 \mathrm{sec}$ of the short-safety and long-safety intervals for the last four days of $70 \mathrm{~V}-140 \mathrm{~V}$ phase were 20.3 and 20.0 , respectively, the difference being virtually none. The corresponding values for the last four days of $80 \mathrm{~V}-160 \mathrm{~V}$ phase were 20.4 and 19.5 for the short-safety and long-safety intervals, respectively, the difference being again nonsignificant $(t=0.75, d f=11)$. The mean number of licks per $5 \mathrm{sec}$ for the successive periods within two different intervals during the last four days of $80 \mathrm{~V}$ - 
$160 \mathrm{~V}$ phase are graphically shown in Fig. 4. As a whole the present results did not give support to the hypothesis that rats would predict the short- and long-safety intervals on the basis of the cues provided by the shock.

\section{Concluding Remarks}

It has been a fairly strong conviction of the present senior author (HI) that the rats would come to modify their behavior in such a way that it would correspond with the regularities present in the environment. Although at the face of them, three experiments reported in the present paper seem to have lacked organic relations with each other, it should be pointed out all these experiments were run with the broad idea of the above kind in mind.

In a licking situation where the shocks were given regularly with the FT schedule, there was clear evidence of temporal conditioning, rats licking less and less as the next shock approached (Imada \& Okamura, 1975). This was also shown in all of the present experiments. Davis et al. (1975) showed in a lever-press situation, in which three shocks were regularly given, that rats' suppression of lever-press recovered remarkably after the third shock was given whenever in a session the third shock occurred. This was the evidence that rats could actually count the number of shocks at least up to three. Rats behaved as though they knew "if three

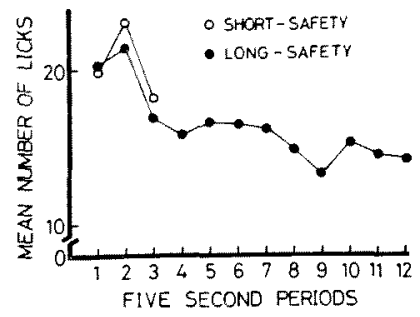

FIG. 4. Mean number of licks in successive $5 \mathrm{sec}$ periods during the short-safety and longsafety intervals. shocks, no more shocks." Naive questions are then that why rats, which are "clever enough to count the number" could not show a DA patterning of licking (Experiments I and II) and also why they could not use the shock-pattern cue to predict the short- and long-safety intervals.

Although it would be too early, on the basis of the present experimental results alone, to draw a conclusion regarding the rats' inability to use shock-cues to predict the forthcoming event and more works have to be definitely done, it was the intention of the present authors to report at this stage that their attempts done so far in pursuing the above possibility have been unsuccessful. As students of psychology of learning, the present authors would like further to investigate the rats' potential ability to use the various subtle cues effectively in predicting danger and safety, before any easy reliance upon such terms like "biological constraints" and "biological boundaries" are made for explanatory purposes. It is hoped that the present series of experiments would contribute to the advancement in this general area of studies.

\section{REFERENCES}

Amsel, A., \& Maltzman, I. 1950 The effect upon generalized drive strength of emotionality as inferred from the level of consummatory response. Fournal of Experimental Psychology, 40, 563-569.

Bloom, J. M., \& Capaldr, E. J. 1961 The behavior of rats in relation to complex patterns of partial reinforcement. Fournal of Comparative and Physiological Psychology, 54, 261-265.

Capald, E.J. 1971 Memory and learning: A sequential viewpoint. In W.K. Honig \& P.H.R. James (Eds.), Animal memory. New York: Academic Press.

Davis, H., Memmotr, J., \& Hurwitz, H.M.B. 1975 Autocontingency: A model for subtle behavioral control. Journal of Experimental Psychology: General, 104, 169-188.

Estes, W. K., \& Skinner, B. F. 1941 Some 
quantitative properties of anxiety. Fournal of Experimental Psychology, 29, 390-400.

Hendry, D. P., Yarczowier, M., \& Switalski, R. C. 1969 Periodic slock with added clock. Journal of the Experimental Analysis of Behavior, 12, 159-166.

Hutchinson, R. R., \& EmLIx, G.S. 1972 Schedule-independent factors contributing to schedule-induced phenomenon. In R. M. Gilbert \& J. D. Keehn (Eds.), Schedule offects: drugs, drinking, and aggression. Toronto \& Buffalo: University of Toronto Press.

Imada, H., \& Okamura, M. 1975 Some cues rats can use as predictors of danger and safety. Animal Leaming and Behavior, 3, 221-225.

Nageishi, Y., \& Imada, H. 1974 Supplession of licking behavior in rats as a function of predictability of shock and probability of conditioned-stimulus-shock pairings. Fournal of Comparative and Ploysiological Psychology, 87, $1165-1173$.
Pavlik, W. B., \& Collier, A. C. 1975 An analysis of "signaled double-alternation patterning" in the rat. Animal Learning and Behavior, 3, 73-75.

Seligman, M.E.P. 1968 Chronic fear produced by unpredictable clectric shock. Fournal of Comparative and Physiological Psychology, 66, $402-411$.

Siecel, P. S, \& Brantley, J.J. 1951 The relationship of emotionality to the consummatory response of eating. Joumal of Experimental Psycliology, 42, 304-306.

Ullman, A. D. 1951 The experimental production and analysis of a "compulsive eating symptom" in rats. Fournal of Comparative and Physiological Psychology, 44, 575--581.

Weiss, K. M., \& Strongman, K. T. 1969 Shockinduced response bursts and suppression. Psychonomic Science, 15, 238-240.

(Received July 17, 1978) 